\title{
THE PLANE FIGURE OF SEVEN REAL LINES*
}

\author{
BY H. S. WHITE
}

1. Introduction. A set of real lines, finite in number, in the plane of projective geometry, divides the plane in to convex polygons, each bounded by segments of those lines. If no three lines meet in a point, then three lines form 4 polygons (triangles); four lines, 7 polygons; $n$ lines, $\left(n^{2}-n+2\right) / 2$ polygons. For any given diagram of this sort, the triangles, quadrilaterals, etc., may be counted; then if the lines are allowed to move freely in the plane, every polygon will retain the same number of sides until three or more points of intersection (or two pairs) come to coincide. Exclude this situation, and we have as invariants the integers showing the numbers of polygons of $3,4,5, \cdots, n$ sides; also a scheme showing contiguities. We shall exhibit such schemes for a set of 7 lines; and inquire how many kinds of (nonequivalent) sets exist, when a one-to-one relation between lines and polygons of two sets constitutes equivalence.

2. Unique Sets, $n=3,4,5$. Since central projection is a particular kind in the group of transformations that we here admit, and since four lines in a plane are projective to any other set of four-barring cases where three lines are copunctual-the arrangement of any such set is typical of all. Three lines form four triangles; three of them have infinitely long boundaries, but that is projectively of no account; and each is adjacent to all the others. A fourth line intersects three segments exterior to one of these triangles, say $T_{1}$, and divides each of the other triangles into a quadrilateral adjacent to $T_{1}$ and a triangle having no sides, but only one vertex, in common with $T_{1}$. Otherwise stated (Fig. 1), four lines in a plane, no three in any point, constitute twelve segments bounding four triangles and three convex quadrilaterals. Each quadrilateral is adjacent to all four triangles, but two triangles have in common only one vertex, while two quadrilaterals have in common two opposite vertices.

Five lines are not necessarily projective to an arbitrarily se-

* Presented to the Society, October 31, 1931. 
lected set of five, but they are equivalent under the group here considered. For a fifth line intersects the four above mentioned upon four segments, each of which separates a triangle from a quadrilateral. Hence the fifth line crosses two triangles and two quadrilaterals; say in cyclic order $T_{1} Q_{1} T_{2} Q_{2}$. The two sides of $T_{1}$ and the two of $T_{2}$ which it meets contain all 5 termini (ver-

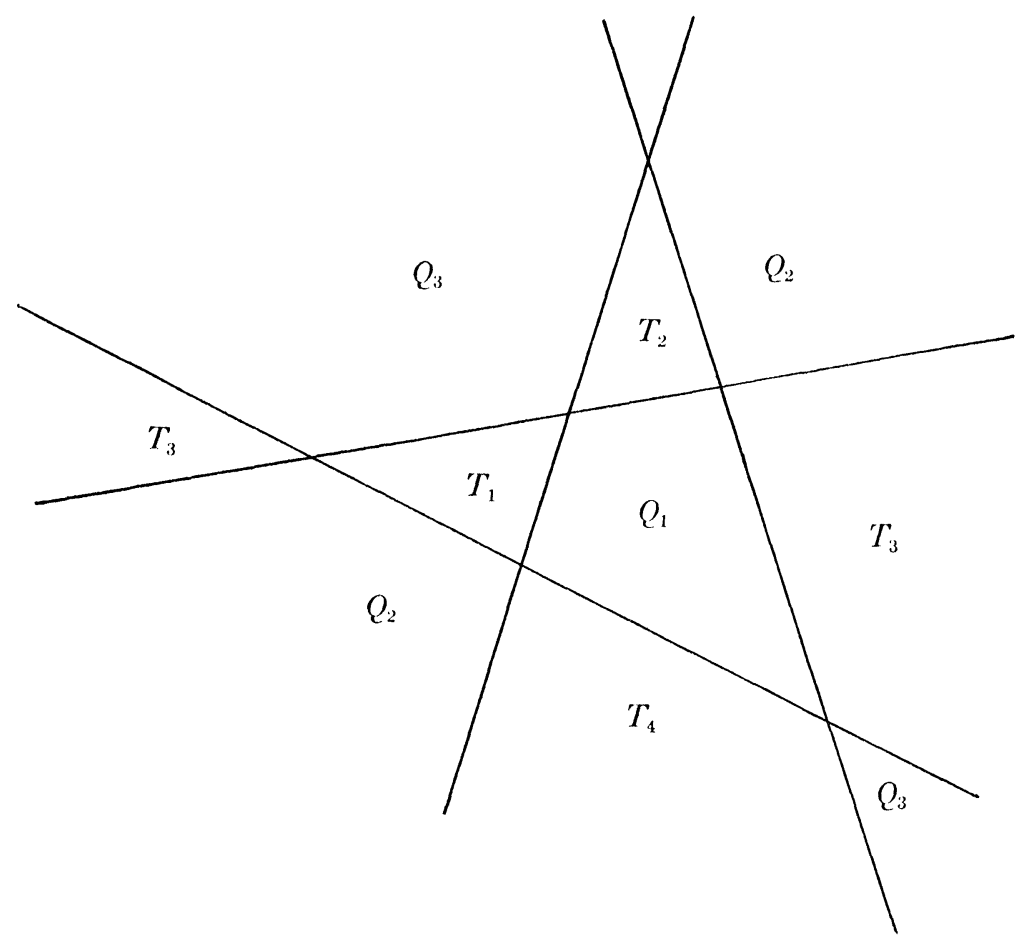

Fig. 1. Lines 4, Quadrilaterals 3, Triangles 4.

tices); hence two sides, one in each triangle, contain the common vertex. But those two must be adjacent sides of either $Q_{1}$ or $Q_{2}$, say of $Q_{1}$; and the other two segments cut by the fifth line have no terminus in common, and are therefore opposite sides of $Q_{2}$. Accordingly $Q_{2}$ is divided into two quadrilaterals, and $Q_{1}$ into a triangle and a pentagon. The triangles $T_{1}$ and $T_{2}$ are divided, each in to a triangle and a quadrilateral.

Among the 11 convex polygons whose boundaries, but no interior 
points, lie in five straight lines of a plane with 10 distinct intersections, there are

1 pentagon;

5 triangles, each adjacent to the pentagon; and

5 quadrilaterals, each adjacent to two triangles and adjacent to two other quadrilaterals.

This description is most easily visualized by the aid of a regular pentagon with sides produced indefinitely. In such a figure all 10 intersections are in the finite plane.

For convenience of allusion, the 20 segments of the 5 lines may be assigned to 3 classes, viz.:

5 primary, separating the pentagon from triangles,

10 secondary, separating the triangles from quadrilaterals,

5 tertiary, separating each, two quadrilaterals.

Note also that each of the 3 classes forms a single continuous broken line, two of five parts, both of even character, one of ten, of odd character. Each quadrilateral has two adjacent sides secondary segments; and two tertiary, adjacent.

3. More than Five Lines. By selecting any five lines of a set as the initial five, we have a unique description for their relative situation. Adding a sixth line, we shall find four different arrangements possible. When there are seven in a set, there are five to be selected at random, and afterward reasons may be found for discriminating the sixth from the seventh. From the diagram of any five lines we may safely write down two observations:

I. As no two triangles have a side in common, no sixth line can cross three triangles. It can cross either two or one.

II. There are lines that meet only tertiary segments, crossing no triangle.

Hence a sixth line will fall into one of the following 4 species (see Fig. 2).

(a) A line cutting 2 primary segments, 2 secondary, 1 tertiary. This species will be divided according as the primary segments are consecutive or not, on the pentagon boundary.

(b) A line cutting 4 secondary segments and 1 tertiary. The two triangles must have one vertex in common.

(c) A line cutting 2 secondary and 3 tertiary segments. This crosses one triangle and four quadrilaterals. 
(d) A line cutting 5 tertiary segments. (See II above.) $a d$ (a) In Fig. 2, line $a$ is shown cutting consecutive sides of the pentagon, thus forming one hexagon.

$a d$ (b) Choosing line $x$ for the sixth or secant line, and pqrsb for the pentagon, we have a secant cutting non-consecutive sides of the pentagon, a case under (a).

$a d(\mathrm{c})$ Exchange roles of $x$ and $c$. Again case (a).

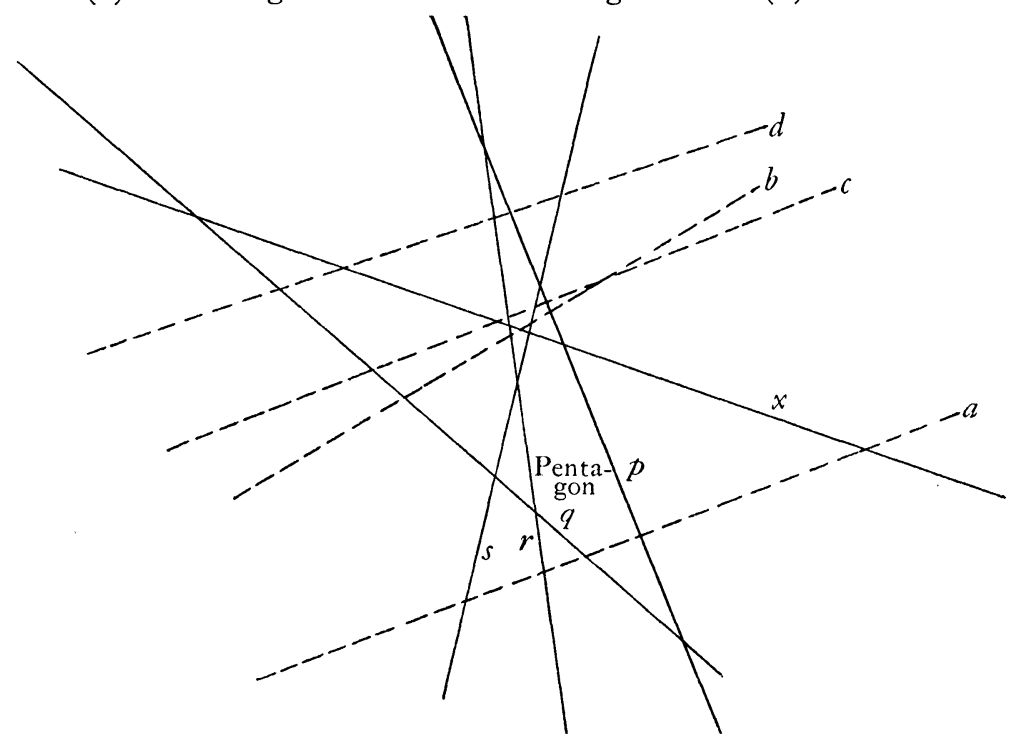

Fig. 2

$a d(\mathrm{~d})$ The sixth line cuts adjacent sides in each of the 5 quadrilaterals, forming 5 new pentagons. Every segment is now a side of some one pentagon. Therefore any seventh line will necessarily cross 3 pentagons, and a new choice of the initial five lines and the sixth will reduce this to case (a).

Incidentally we may tabulate the sets of polygons formed by five or six lines, convex polygons having no points of the lines in their interior.

Polygons of

Five lines

Six lines

\begin{tabular}{l|r|r|r|r|} 
& \multicolumn{1}{c}{6} & 5 & 4 & 3 \\
\hline & 0 & 1 & 5 & 5 \\
$\mathrm{a}$ & 1 & 0 & 9 & 6 \\
$\mathrm{~b}$ & 0 & 2 & 8 & 6 \\
$\mathrm{c}$ & 0 & 3 & 6 & 7 \\
$\mathrm{~d}$ & 0 & 6 & 0 & 10 \\
\hline
\end{tabular}

sides formed by as in Figure 2. 
Division of the subject. When, in a set of seven lines, any six form a hexagon, the discussion may utilize that hexagon as unique initial figure. This part of the subject has been investigated by Miss L. D. Cummings. I shall survey only sets of seven lines in which no six form a convex hexagon.

4. Notation and Method. Let there be given a pentagon and a secant crossing two segments of its boundary, not coterminous (consecutive), since then a hexagon would be formed. This secant must cross one and only one tertiary segment at a point which we shall call $A$, and two secondary segments belonging to two triangles. Of those triangles, one or both must have seg-

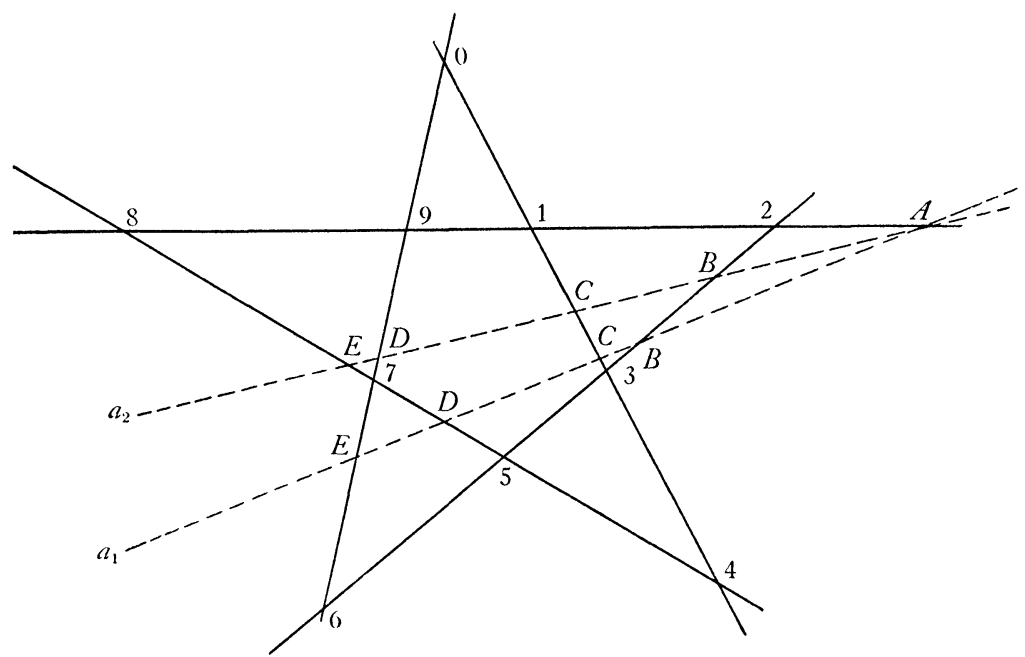

FIG. 3

ments in their boundary collinear with that unique tertiary segment, since otherwise their four secondary boundaries would lie in the other four lines of the pentagon and the two triangles would have one vertex in common, whence the primary sides would be consecutive. Call the secant line $a_{1}$ when it crosses only one triangle with a side collinear to $A$; call it $a_{2}$ when it crosses both triangles having secondary sides collinear with $A$. (See Fig. 3.)

The Secant is $a_{1}$. Designate by 12 the secondary side, collinear with $A$, of the first triangle crossed by the secant; by 23 its other 
secondary side, crossing the secant at $B$. Mark the other intersections of the secant so that the five are in order on that secant, $A B C D E$. Two vertices of the pentagon are already marked; let the five vertices in order on the boundary of the pentagon be $1,3,5,7,9$. On the broken line of secondary segments insert 4,6 , 8,0 in due order between odd digits. Now arrange in continuous broken-line divisions the segments that cross the secant, beginning with any point terminating only one such segment. Thus the secant $a_{1}$ will be recorded by 8231.576 .

The Secant is $a_{2}$. Proceed in the same way. The choice of point 1 is in this case ambiguous, and there are two admissible sets of marks. If the one set is

$$
\text { 1234567890ABCDE, }
$$

then the other set is

$$
9876543210 A E D C B \text {. }
$$

We may call this the symmetric case, the other unsymmetric. For $a_{2}$, the recorded position will be 978231 .

It remains to form all possible records for a seventh line which shall not cut consecutive sides of this pentagon, using both numerals and letters. Every set of seven lines can be separated in 21 ways in to five-and-two; then whenever both lines of the two cross the pentagon of the others, two records will be permitted by exchange of sixth and seven th line roles. Conceivably, therefore, 42 records might denote a single set of 7 lines. Always there will be at least one record, since at least one choice of five lines from the seven will leave a secant $a_{1}$ or $a_{2}$.

5. Experimental or Observational Geometry. Since a definite order is essential to an exhaustive census, I have taken small sets of positions for the seventh line; five for case $a_{1}$, three for case $a_{2}$ (symmetric), collecting together those that cut the same segment of the sixth line. Each position is derived from one already obtained (starting from coincidence with the sixth secant) by sweeping over one of the ten vertices of the complete pentagon. This is not complicated, and after the list is filled out for each segment, $A B, B C, \cdots$, then I erase all that show two consecutive segments from the boundary of either pentagonthe fundamental one; or, for $a_{1}$, that second one with vertices $A B 348$; or the third, $C D 791$. In this way reduced lists are 
fixed. For example, in case $a_{1}$, where the sixth secant designated by 8231.576 , if the seventh crosses the segment $A B$, it may be any one of the following:

$\begin{array}{lll}2 A B .3456 E & 62 A B .109 .84 & 6048 . A B 21 \\ B A 260.345 & 4897 . C 1 . A B 2 & 60.345 . A B 21 \\ 84062 A B & 48 . A B 2109 & 840.56 E .2 A B .\end{array}$

For each of these in turn 21 duplicate diagrams are made, beginning with the first. Each diagram has a pentagon and two secants outlined in colored crayon. When hexagons appear, six or ten to any set, or in one case the heptagon, those are laid aside; as are also the sketches in which both secants are external to the pentagon (cases (c) or (d)). For each sketch not eliminated, either one or two records are determined on the basis of $\S 4$, and these are checked off on the list and classed as equivalents.

By this means, three systems and no more are found in which no hexagon occurs. Representative descriptions of those three are the following:

\subsection{6 and $62 A B .109 .84,8231.576$ and $2 A B .3456 E$, 8231.576 and $84062 A B$.}

This work though laborious is not tedious. Mistakes and omissions occur, but the multitude of equivalences makes their discovery almost certain. Aside from the intrinsic interest of such a catalog, we have undertaken the problem for the purpose of studying Aronhold sets of seven double-tangents of a plane quartic curve. As to polygonal division of the projective plane by lines, we note for $n=5,6$, or 7 the contrast between our results and the much larger number of systems that satisfy Euler's equation. The polygons in these three non-hexagonal sets of seven lines are as follows, in order as above.

\begin{tabular}{|c|r|r|r|r|r|}
\hline No. of sides & 7 & 6 & 5 & 4 & 3 \\
\hline 1 & 0 & 0 & 3 & 12 & 7 \\
2 & 0 & 0 & 4 & 10 & 8 \\
3 & 0 & 0 & 6 & 6 & 10 \\
\hline
\end{tabular}

Vassar College 